\title{
A CONVOLUTION APPROACH TO CERTAIN SUBCLASSES OF STARLIKE FUNCTIONS
}

\author{
T. RAM REDDY, O. P. JUNEJA AND K. SATHYANARAYANA
}

\begin{abstract}
The class $R_{\gamma}(A, B)$ for $-1 \leq B<A \leq 1$ and $\gamma>(A-1) /(1-B)$ consisting of normalised analytic functions in the open unit disc is defined with the help of Convolution technique. It consists of univalent starlike functions for $\gamma \geq 0$. We establish containment property, integral transforms and a sufficient condition for an analytic function to be in $R_{\gamma}(A, B)$. Using the concept of dual spaces we find a convolution condition for a function in this class.
\end{abstract}

\section{Introduction.}

Let $H$ denote the class of functions $f(z)=z+a_{2} z^{2}+\ldots$ that are analytic in the unit disc $E=\{z /|z|<1\}$. Let $S$ denote the subclass of $H$ consisting of univalent functions. Let $S_{\beta}$ and $K_{\beta}(0 \leq \beta<1)$ denote the classes of functions that are starlike of order $\beta$ and convex of order $\beta$ respectively, $S_{0}=S$ and $K_{0}=K$ are wellknown classes of starlike (with respect to origin) and convex functions respectively. The Convolution or Hadamard product of two power series $f(z)=\sum_{n=0}^{\infty} a_{n} z^{n}$ and $g(z)=\sum_{n=0}^{\infty} b_{n} z^{n}$ is defined as the power series $(f g)(z)=\sum_{n=0}^{\infty} a_{n} b_{n} z^{n}$.

Let $A, B$ and $\gamma$ be arbitrary fixed real numbers such that $-1 \leq B<A \leq 1$ and $\gamma>(A-1) /(1-B)$, we say that a function $f$ in $H$ is in the class $R_{\gamma}(A, B)$ if it satisfies the condition

$$
\frac{z\left(D^{\gamma} f(z)\right)^{\prime}}{D^{\gamma} f(z)} \ll \frac{1+A z}{1+B z}, \quad z \in E .
$$

where' denotes the differentiation with respect to $z$, « denotes the subordination and $D^{\gamma} f(z)=z /(1-z)^{\gamma+1} * f(z)$. Let $B_{0}$ denote the class of analytic functions $w(z)$ in $E$ such that $w(0)=0$ and $|w(z)|<|z|$. From the subordination principle, the equation (1.1) becomes,

$$
\frac{z\left(D^{\gamma} f(z)\right)^{\prime}}{D^{\gamma} f(z)}=\frac{1+A w(z)}{1+B w(z)} \text { for some } w(z) \in B_{0} \text {. }
$$

Received January 23, 1991.

1980 Mathematics subject classification (1985 Revision), Primary 30 C 45, Secondary 30 C 55. 
It can be seen that

$$
z\left(D^{\gamma} f(z)\right)^{\prime}=(\gamma+1) D^{\gamma+1} f(z)-\gamma D^{\gamma} f(z)
$$

From this, the equation (1.2) is equivalent to

$$
\frac{D^{\gamma+1} f(z)}{D^{\gamma} f(z)}=\frac{\gamma}{\gamma+1}+\frac{1}{\gamma+1} \frac{1+A w(z)}{1+B w(z)}
$$

We observe that if a function $f(z)$ is in $R_{\gamma}(A, B)$ then it satisfies

$$
\left|\frac{z\left(D^{\gamma} f(z)\right)^{\prime}}{D^{\gamma} f(z)}-\frac{1-A B}{1-B^{2}}\right|<\frac{A-B}{1-B^{2}} \text { for } z \in E, B \neq-1 .
$$

and

$$
\operatorname{Re}\left\{\frac{z\left(D^{\gamma} f(z)\right)^{\prime}}{D^{\gamma} f(z)}\right\}>\frac{1-A}{2} \text { for } z \in E, B=-1 .
$$

The class $R_{\gamma}(A, B)$ has been introduced and studied by the first author [4]. It can be easily seen that $R_{0}(A, B)=S^{*}(A, B)$ and $R_{1}(A, B)=K(A, B)$ which are respectively the subclasses of starlike and convex functions considered by Janowski [3].

It is observed that for particular choices of the parameters involved in $R_{\gamma}(A, B)$ we get the following classes: For $0 \leq \alpha<1$ and $0 \leq \beta<1, R_{1-2 \alpha}(1-2 \beta,-1)(\equiv R(\alpha, \beta)$ in the notation of [9]) has been introduced and studied by Sheil-Small, Silverman and Silvia [9] and for any $\alpha(0 \leq \alpha<1)$ and $\gamma=n \in N_{0}$ (a non-negative integer) we have $R_{n}(1-2 \alpha,-1)=R_{n}(\alpha)$ which was studied recently by Ahuja and Silverman [1].

In the present paper we shall establish the containment property $R_{\gamma+1}(A, B) \subset$ $R_{\gamma}(A, B)$ for $-1 \leq B<A \leq 1$ and $\gamma>(A-1) /(1-B)$ and hence $R_{\gamma}(A, B)$ consists of univalent functions for atleast $\gamma \geq 0$. We also study some other aspects such as integral transforms, sufficient condition and convolution properties of functions belonging to the class $R_{\gamma}(A, B)$. These results generalize and improves the recent results of Ahuja and Silverman [1], Sheil-Small, Silverman and Silvia [9] and Ram Singh and Sunder Singh $[5]$.

\section{Contaimment Properties}

The following theorem gives the characterization of the class $R_{\gamma}(A, B)$.

Theorem 2.1. $R_{\gamma+1}(A, B) \subset R_{\gamma}(A, B)$ holds for $-1 \leq B<A \leq 1$ and $\gamma>$ $(A-1) /(1-B)$.

Proof. Suppose $f(z)$ is in $R_{\gamma+1}(A, B)$, then we have

$$
\left|\frac{z\left(D^{\gamma+1} f(z)\right)^{\prime}}{D^{\gamma+1} f(z)}-\frac{1-A B}{1-B^{2}}\right|<\frac{A-B}{1-B^{2}} \text { for } z \in E, B \neq-1 \text {. }
$$




$$
\operatorname{Re}\left\{\frac{z\left(D^{\gamma+1} f(z)\right)^{\prime}}{D^{\gamma+1} f(z)}\right\}>\frac{1-A}{2}, \text { for } z \in E, B=-1
$$

Define a function $w(z)$ such that

$$
\frac{z\left(D^{\gamma} f(z)\right)^{\prime}}{D^{\gamma} f(z)}=\frac{1+A w(z)}{1+B w(z)}
$$

The function $w(z)=\left[\frac{z\left(D^{\gamma} f(z)\right)^{\prime}}{D^{\gamma} f(z)}-1\right]\left[A-B \frac{z\left(D^{\gamma} f(z)\right)^{\prime}}{D^{\gamma} f(z)}\right]^{-1}$ with $w(0)=0, w(z) \neq 1$ is either meromorphic or regular in $E$. It is sufficient to show that $|w(z)|<1$ for $z \in E$. Using the identity (1.3), the equation (2.3) reduces to

$$
\frac{D^{\gamma+1} f(z)}{D^{\gamma} f(z)}=\frac{\gamma}{\gamma+1}+\frac{1}{\gamma+1} \frac{1+A w(z)}{1+B w(z)} .
$$

Differentiating (2.4) logarithmically and using (2.3) and (1.3) we obtain

$$
\frac{z\left(D^{\gamma+1} f(z)\right)^{\prime}}{D^{\gamma+1} f(z)}=\frac{1+A w(z)}{1+B w(z)}+\frac{(A-B) z w^{\prime}(z)}{[(\gamma+1)+(A+B \gamma) w(z)][1+B w(z)]}
$$

Case (i). If $B \neq-1$, then we have from (2.5),

$$
\begin{aligned}
& \frac{z\left(D^{\gamma+1} f(z)\right)^{\prime}}{D^{\gamma+1} f(z)}-\frac{1-A B}{1-B^{2}} \\
= & \frac{A-B}{1-B^{2}} \frac{B+w(z)}{1+B w(z)}+\frac{\left(1-B^{2}\right) z w^{\prime}(z)}{[(\gamma+1)+(A+B \gamma) w(z)][1+B w(z)]}
\end{aligned}
$$

Let $z_{1}$ with $\left|z_{1}\right|=r_{1}$ be the pole of $w(z)$ in $E$ that is nearest to the origin. Hence $w(z)$ is regular in $|z| \leq r_{1}<1$. By Jack's lemma [2], we have for $|z| \leq r<r_{1}$ there is a point $z_{0}$ such that

$$
z_{0} w^{\prime}(z)=k w\left(z_{0}\right), \quad k \geq 1
$$

At this point $z_{0}$, using (2.7) the equation (2.6) reduces to

$$
\begin{gathered}
\frac{z_{0}\left(D^{\gamma+1} f\left(z_{0}\right)\right)^{\prime}}{D^{\gamma+1} f\left(z_{0}\right)}-\frac{1-A B}{1-B^{2}}=\frac{T\left(z_{0}\right)}{R\left(z_{0}\right)} \frac{A-B}{1-B^{2}}, \text { where } \\
T\left(z_{0}\right)=d+h w\left(z_{0}\right)+g w^{2}\left(z_{0}\right)
\end{gathered}
$$

and

$$
R\left(z_{0}\right)=(\gamma+1)+(d+g) w\left(z_{0}\right)+\frac{d g}{\gamma+1} w^{2}\left(z_{0}\right) \text { with }
$$

$d=B(\gamma+1), h=(\gamma+1)+B(A+B \gamma)+k\left(1-B^{2}\right)$ and $g=A+B \gamma$ 
Now suppose that it was possible to have $M(r, w)=\operatorname{Max}|w(z)|=1$, for some $r<r_{1}<1$. At the point $z_{0}$ where this occured we would have $|w(z)|=1$, then

$$
\left|T\left(z_{0}\right)\right|^{2}=d^{2}+h^{2}+g^{2}+2(d+g) h \operatorname{Re} w\left(z_{0}\right)+2 d g \operatorname{Re} w^{2}\left(z_{0}\right)
$$

and

$$
\begin{aligned}
\left|R\left(z_{0}\right)\right|^{2} & =(\gamma+1)^{2}+(d+g)^{2}+\left(\frac{d g}{\gamma+1}\right)^{2}+2\left[(\gamma+1)+\frac{d g}{\gamma+1}\right] \\
& \times(d+g) \operatorname{Re} w\left(z_{0}\right)+2 d g \operatorname{Re} w^{2}\left(z_{0}\right) .
\end{aligned}
$$

From (2.11) and (2.12) we have

$$
\left|T\left(z_{0}\right)\right|^{2}-\left|R\left(z_{0}\right)\right|^{2}=E+2 F \operatorname{Re} w\left(z_{0}\right)
$$

where

$$
\begin{aligned}
E & =d^{2}+h^{2}+g^{2}-\left[(\gamma+1)^{2}+(d+g)^{2}+\left(\frac{d g}{\gamma+1}\right)^{2}\right] \\
& =k\left(1-B^{2}\right)\left[k\left(1-B^{2}\right)+2\{(\gamma+1)+B(A+B \gamma)\}\right] \text { and } \\
F & =(d+g) h-\left[(\gamma+1)+\frac{d g}{\gamma+1}(d+g)\right]=B[(\gamma+1)+A+B \gamma] k\left(1-B^{2}\right) .
\end{aligned}
$$

Thus

$$
\left|T\left(z_{0}\right)\right|^{2}-\left|R\left(z_{0}\right)\right|^{2} \geq 0 \text { provided } E \pm 2 F \geq 0 .
$$

Now $E+2 F=k\left(1-B^{2}\right)\left[k\left(1-B^{2}\right)+2\{(\gamma+1)+A+B \gamma\}(1+B)\right]>0$, since $\gamma>(A-1) /(1-B)$.

Also $E-2 F=k\left(1-B^{2}\right)\left[k\left(1-B^{2}\right)+2\{(\gamma+1)-(A+B \gamma)\}(1+B)\right]>0$, since $\gamma>(A-1) /(1-B)$.

From (2.8) and (2.14) we have

$$
\left|\frac{z_{0}\left(D^{\gamma+1} f(z)\right)^{\prime}}{D^{\gamma+1} f(z)}-\frac{1-A B}{1-B^{2}}\right| \geq \frac{A-B}{1-B^{2}} .
$$

which is a contradiction to (2.1). So we cannot have $\max _{|z|<r_{1}}|w(z)|=1$. Thus $|w(z)| \neq 1$ in $|z|<r_{1}$. Since by our assumption $w(z)$ is analytic in the disk $|z|<r_{1},|w(z)|$ is continuous there. Again since $w(0)=0$ and $|w(z)| \neq 1$. hence $w(z)$ cannot have a pole at $|z|=r_{1}$. As $r_{1}$ is arbitrary. $w(z)$ is analytic in $E$ and $|w(z)|<1$. Hence $f(z)$ is in $R_{\gamma}(A, B)$.

Case (ii). If $B=-1$, then (2.5) reduces to

$$
\frac{z\left(D^{\gamma+1} f(z)\right)^{\prime}}{D^{\gamma+1} f(z)}=\frac{1+A w(z)}{1+B w(z)}+\frac{(A+1) z w^{\prime}(z)}{\{(\gamma+1)+(A-\gamma) w(z)\}(1-w(z))}
$$


We claim again that $|w(z)|<1$. Suppose if possible that there is a point $z_{0}$ in $E$ such that $\max _{|z| \leq\left|z_{0}\right|}|w(z)|=\left|w\left(z_{0}\right)\right|=1$, then by Jack's lemma [2], we have $z_{0} w^{\prime}\left(z_{0}\right)=$ l. $w\left(z_{0}\right)$, $k \geq 1$. Hence at the point $z_{0}$ with $w\left(z_{0}\right)=e^{i \Phi}(0 \leq \Phi<2 \pi)$ we have from (2.15)

$$
\begin{aligned}
& \operatorname{Re}\left\{\frac{z_{0}\left(D^{\gamma+1} f\left(z_{0}\right)\right)^{\prime}}{D^{\gamma+1} f\left(z_{0}\right)}\right\} \\
= & \operatorname{Re}\left\{\frac{1+A e^{i \Phi}}{1-e^{i \Phi}}\right\}+\frac{(1+A) k e^{i \Phi}}{\left\{(\gamma+1)+(A-\gamma) e^{i \Phi}\right\}\left(1-e^{i \Phi}\right\}} \\
= & \frac{1-A}{2}+k\left[-\frac{1}{2}+\frac{(A-\gamma)^{2}+(A-\gamma)(\gamma+1) \cos \Phi}{(\gamma+1)^{2}+(A-\gamma)^{2}+2(\gamma+1)(A-\gamma) \cos \Phi}\right] \\
= & (1-A) / 2, \quad \text { since } k \geq 1 \text { and } \gamma>(A-1) / 2 .
\end{aligned}
$$

which is again a contradiction to (2.2). Thus we must have $|w(z)|<1$. Hence the theorem is true for $B=-1$ also. This completes the proof.

\section{Integral Transforms}

In this section we consider certain Bernadi type of integral transforms in the flass $R_{\gamma}(A, B)$.

Theorem 3.1. If $g(z)$ is in $R_{\gamma}(A, B), \gamma \geq 0$ and $G(z)$ is defined by

$$
G(z)=(1+c) \int_{0}^{1} u^{c-1} g(u z) d u, \text { for } c>(A-1) /(1-B)
$$

then $G(z)$ is in $R_{\gamma}(A, B)$.

Proof. Suppose $g(z)$ is in $R_{\gamma}(A, B)$, then we have

$$
\left|\frac{z\left(D^{\gamma} g(z)\right)^{\prime}}{D^{\gamma} g(z)}-\frac{1-A B}{1-B^{2}}\right|<\frac{A-B}{1-B^{2}} \quad \text { if } B \neq-1, \text { for } z \in E \text {. }
$$

and

$$
\operatorname{Re}\left\{\frac{z\left(D^{\gamma} g(z)\right)^{\prime}}{D^{\gamma} g(z)}\right\}>(1-A) / 2 \text { if } B=-1 \text { for } z \in E .
$$

From the definition of $G(z)$, we have

$$
z G^{\prime}(z)+c G(z)=(1+c) g(z)
$$

Taking convolution on both sides of (3.4) with $\frac{z}{(1-z)^{\gamma+1}}$ for $\gamma \geq 0$, we get

$$
z\left(D^{\gamma} G(z)\right)^{\prime}+c D^{\gamma} G(z)=(1+c) D^{\gamma} g(z)
$$


where we have made use of the fact that $D^{\gamma}\left(z G^{\prime}(z)\right)=z\left(D^{\gamma} G(z)\right)^{\prime}$. Thus

$$
\frac{z\left(D^{\gamma} G(z)\right)^{\prime}}{D^{\gamma} G(z)}+c=(1+c) \frac{D^{\gamma} g(z)}{D^{\gamma} G(z)}
$$

since $h(z)=\frac{z\left(D^{\gamma} G(z)\right)^{\prime}}{D^{\gamma} G(z)}$ is analytic at $z=0$, define a function $w(z)$ such that

$$
\frac{z\left(D^{\gamma} G(z)\right)^{\prime}}{D^{\gamma} G(z)}=\frac{1+A w(z)}{1+B w(z)} .
$$

The function $w(z)=\left[\frac{z\left(D^{\gamma} G(z)\right)^{\prime}}{D^{\gamma} G(z)}-1\right]\left[A-B \frac{z\left(D^{\gamma} G(z)\right)^{\prime}}{D^{\gamma} G(z)}\right]^{-1}$ with $w(0)=0, w(z) \neq 1$ is either analytic or meromorphic in $E$. It is sufficient to show that $|w(z)|<1$ for $z \in E$.

From (3.6) and (3.7) we have

$$
\frac{D^{\gamma} g(z)}{D^{\gamma} G(z)}=\frac{1}{1+c}\left[\frac{(1+c)+(A+B c) w(z)}{1+B w(z)}\right]
$$

Differentiating (3.8) logarithmically, using (3.7) and (1.3) we have

$$
\frac{z\left(D^{\gamma} g(z)\right)^{\prime}}{D^{\gamma} g(z)}=\frac{1+A w(z)}{1+B w(z)}+\frac{(A-B) z w^{\prime}(z)}{[(1+c)+(A+B c) w(z)][1+B w(z)]}
$$

We observe that the right hand side of (3.9) is essentially same as that of (2.5) except that $\gamma$ is replaced by $c$ in the second term. Thus proceeding exactly in similar manner as in the proof of Theorem 2.1, analogous argument gives that $|w(z)|<1$ for $z \in E$. Therefore $G(z) \in R_{\gamma}(A, B)$.

When $A=1, B=-1$ and $\gamma \in N_{0}$ we obtain a result due to Ram Singh and Sunder Singh [5].

Corollary 3.1(a). If $g(z)$ is in $R_{n}(1,-1)$, then the function $G(z)$ defined by (3.1) is also in $R_{n}(1,-1)$.

Again putting $A=1-2 \beta(0 \leq \beta<1), B=-1$ and $\gamma=1-2 \alpha$ in Theorem 3.1 we obtain the following corollary that seems to be a new result for the class $R_{1-2 \alpha}(1-$ $2 \beta,-1$ ).

Corollary 3.1(b). Let $g(z)$ be in $R_{1-2 \alpha}(1-2 \beta,-1)$ then $G(z)$ defined by (3.1) is in $R_{1-2 \alpha}(1-2 \beta,-1)$.

The Theorem 3.1 can be further strengthened if one takes $\gamma$ and $c$ to be non negative integers. Thus we obtain.

Theorem 3.2. If $g(z)$ is in $R_{n}(A, B)$ and $G(z)$ is defined by

$$
G(z)=(n+1) \int_{0}^{1} u^{n-1} g(u z) d u, \quad n=0,1,2, \ldots
$$


then $G(z)$ is in $R_{n+1}(A, B)$.

Proof. From the definition of $G(z)$ we have

$$
n G(z)+z G^{\prime}(z)=(n+1) g(z)
$$

or

$$
n D^{n} G(z)+z\left(D^{n} G(z)\right)^{\prime}=(n+1) D^{n} g(z)
$$

Now using the identity (1.3) we have,

$$
\begin{gathered}
D^{n+1} G(z)=D^{n} g(z) \text {. Similarly we have } \\
(n+1) D^{n+1} g(z)=(n+2) D^{n+2} G(z)-D^{n+1} G(z) .
\end{gathered}
$$

From (3.11) and (3.12) we have

$$
\frac{D^{n+1} g(z)}{D^{n} g(z)}=\frac{(n+2) D^{n+2} G(z)-D^{n+1} G(z)}{(n+1) D^{n+1} G(z)}
$$

Since $g(z)$ is in $R_{n}(A, B)$ we have

$$
\frac{D^{n+1} g(z)}{D^{n} g(z)}=\frac{n}{n+1}+\frac{1}{n+1} \frac{1+A w(z)}{1+B w(z)} \text { for some } w(z) \in B_{0} \text {. }
$$

From (3.13) and (3.14) we have

$$
\frac{D^{n+2} G(z)}{D^{n+1} G(z)}=\frac{n+1}{n+2}+\frac{1}{n+2} \frac{1+A w(z)}{1+B w(z)} .
$$

Thus it follows from (3.15) that $G(z)$ is in $R_{n+1}(A, B)$.

Setting $A=1-2 \alpha$ and $B=-1$ we obtain the result due to Ahuja and Silverman [1].

Corollary 3.2. If $g(z)$ is in $R_{n 2}(\alpha)$ then $G(z)$ defined by (3.10) is in $R_{n+1}(\alpha)$.

Remark. This Theorem has been proved in [8] by using different technique.

\section{Sufficient Condition}

We find a sufficient condition for a function $f(z)$ in $H$ to be in $R_{\gamma}(A, B)$.

Theorem 4.1. For a function $f(z)=z+\sum_{n=2}^{\infty} a_{n} z^{n}$ in $H$ such that if for some real number $\gamma \geq 0$ and $A, B$ with $-1 \leq B<A \leq 1$,

$$
\sum_{n=2}^{\infty}\{(n+1)+|A-B n|\} C(\gamma, n)\left|a_{n}\right| \leq A-B \text { holds }
$$


then $f(z)$ belongs to the class $R_{\gamma}(A, B)$, where

$$
C(\gamma, n)=\frac{\prod_{j=2}^{n}(\gamma+j)}{(n-1) !}
$$

Proof. Suppose that (4.1) holds. Since $D^{\gamma} f(z)=z+\sum_{n=2}^{\infty} C(\gamma, n) a_{n} z^{n}$, we have for $z \in E$

$$
\begin{aligned}
& \left|z\left(D^{\gamma} f(z)\right)^{\prime}-D^{\gamma} f(z)\right|-\left|A D^{\gamma} f(z)-B z\left(D^{\gamma} f(z)\right)^{\prime}\right| \\
= & \left|\sum_{n=2}^{\infty}(n-1) C(\gamma, n) a_{n} z^{n}\right|-\left|(A-B) z+\sum_{n=2}^{\infty}(A-B n) C(\gamma, n) a_{n} z^{n}\right| \\
\leq & \sum_{n=2}^{\infty}(n-1) C(\gamma, n)\left|a_{n}\right| r^{n}-\left\{(A-B) r-\sum_{n=2}^{\infty}|A-B n| C(\gamma, n)\left|a_{n}\right|\right\} r \\
= & \left\{\sum_{n=2}^{\infty}[(n-1)+|A-B n|] C(\gamma, n)\left|a_{n}\right|-(A-B)\right\} r \leq 0 \text { by (4.1). }
\end{aligned}
$$

Hence it follows that,

$$
\left[\frac{z\left(D^{\gamma} f(z)\right)^{\prime}}{D^{\gamma} f(z)}-1\right]\left[A-B \frac{z\left(D^{\gamma} f(z)\right)^{\prime}}{D^{\gamma} f(z)}\right]^{-1}<1
$$

Let $w(z)$ denote the term inside the modulus of $(4.3)$ then $w(0)=0, w(z)$ is analytic in $|z|<1$ and $|w(z)|<1$. Hence we have

$$
\frac{z\left(D^{\gamma} f(z)\right)^{\prime}}{D^{\gamma} f(z)}=\frac{1+A w(z)}{1+B w(z)} \text { which shows that }
$$

$f(z)$ belongs to the class $R_{\gamma}(A, B)$.

\section{Convolution Properties and Convolution Condition of $R_{\gamma}(A, B)$}

By making use of the concept of Ruschewcyh and Sheil-Small [7], we have shown in [8] that is $f$ and $g$ are in $R_{\gamma}(A, B)$ then so is $f * g$ for $\gamma \geq 1$. In fact it can be seen that if $f$ is in $K$ and $g$ is in $R_{\gamma}(A, B)$ then $\int * g$ is in $R_{\gamma}(A, B)$ for $\gamma \geq 0$.

To establish the convolution condition for $R_{\gamma}(A, B)$ we use the concept of dual spaces due to Ruscheweyh [6]. Given a normal family $F \subset H$, the dual of $F$, denoted by $F^{*}$ is

$$
\{f \in H / f * g \neq 0 \text { for all } g \in F, \quad 0<|z|<1\} \text {. }
$$

Let $F_{\gamma}(A, B)=\left\{\frac{z+\frac{(\gamma+1) \mu-(A+B \gamma)}{(A-B)} z^{2}}{(1-z)^{\gamma+2}} /|\mu|=1\right\}$ be any normal family in $H$. In [9 and $10]$ they have been pointed out that $S^{*}(A, B)$ and $K(A, B)$ are the duals of $F_{0}(A, B)$ and 
$F_{1}(A, B)$ respectively. In this section we need to establish the condition for a function to be in $R_{y}(A, B)$ interms of these dual spaces.

Theorem 5.1. A function $f(z)$ in $H$ is in the class $R_{\gamma}(A, B)(\gamma \geq 0)$ if and only if $R_{\gamma}(A, B)$ is the dual of $F_{\gamma}(A, B)$.

Proof. Suppose $f(z)$ is in $R_{\gamma}(A, B)$ then $D^{\gamma} f(z)$ is in $S^{*}(A, B)$.

Since $\frac{z\left(D^{\gamma} f(z)\right)^{\prime}}{D^{\gamma} f(z)}=1$ at $z=0$, therefore

$$
\frac{z\left(D^{\gamma} f(z)\right)^{\prime}}{D^{\gamma} f(z)} \neq \frac{1+A x}{1+B x} \text { for some } x \text { with }|x|=1 \text { and } x \neq 1
$$

in $0<|z|<1$. From (5.1) and making use of the identity (1.3) we get

$$
(1+B x)\left\{(\gamma+1) D^{\gamma+1} f(z)-\gamma D^{\gamma} f(z)\right\}-(1+A x) D^{\gamma} f(z) \neq 0
$$

Using $D^{\gamma} f(z)=z /(1-z)^{\gamma+1} * f(z)$ in (5.2) we have

$$
f\left(z *\left[\left\{(\gamma+1) \frac{z}{(1-z)^{\gamma+2}}-\gamma \frac{z}{(1-z)^{\gamma+1}}\right\}(1+B x)-\frac{z}{(1-z)^{\gamma+1}}(1+A x)\right] \neq 0\right.
$$

in $0<|z|<1$, which on simplification reduces to

$$
f(z) * \frac{z(B-A) x+\{(\gamma+1)+(A+B \gamma) x\} z^{2}}{(1-z)^{\gamma+2}} \neq 0 .
$$

since $x \neq 1$ and $B-A$ is negative, putting $\mu=-1 / x$, then the above condition reduces to

$$
f(z) * \frac{z+\{(\gamma+1) \mu-(A+B \gamma)\}(A-B)^{-1} z^{2}}{(1-z)^{\gamma+2}} \neq 0, \quad 0<|z|<1
$$

which is the desired convolution condition. The converse part follows easily since all the steps can be retraced back.

It can be easily seen that for different choices of the values $A=1-2 \beta(0 \leq \beta<1)$, $B=-1$ and $\gamma=1-2 \alpha$ we obtain a convolution condition for the class $R_{1-2 \alpha}(1-2 B,-1)$ of Sheil-Small, Silverman and Silvia [9]. Also for $A=1-2 \alpha(0 \leq \alpha<1), B=-1$ and $\gamma \in N_{0}$ we get the recent convolution condition for the class $R_{n}(\alpha)$ of Ahuja and Silverman [1].

\section{A cknowledgements}

The first author would like to thank U. G. C. (New Delhi) for providing a Minor Research Project and third author is indebted to C. S. I. R. (New Delhi) for awarding a Senior Research Fellowship. 


\section{References}

[1] O. P. Ahuja and H. Silverman, "Function classes related to Ruscheweyh derivatives", J. Austral. Math. Soc., (Series A) 47 (1989), 438-444.

[2] I. S. Jack, "Functions starlike and convex of order $\alpha$ ", J. Lon Math. Soc. (2) 3 (1971), 469-474.

[3] W. Janowski, "Some extremal problems for certain families of analytic functions I", Ann. Polon. Math. 28 (1973), 297-326.

[4] T. Ram Reddy, "A study on certain subclasses of univalent analytic functions", Ph. D. Thesis, I. I. T. Kanpur, 1983.

[5] Ram Singh and Sunder Singh, "Integrals of certain univalent functions", Proc. Amer. Math. Soc., 77 (1979), 336-340.

[6] S. Ruscheweyh, "Duality for Hadamard products with applications to extremal problems for functions regular in the unit disc", Trans. Amer. Math. Soc. 210 (1975), 63-74.

[7] S. Ruscheweyh and T. Sheil-Small, "Hadamard product of schlicht functions and Polya Schoenberg Conjecture", Comment. Math. Helve., 48 (1973), 119-135.

[8] K. Sathyanarayana and T. R. Reddy, "An applications of convolution for a subclasses of starlike functions (Communicated)".

[9] T. Sheil-Small, H. Silverman and E. M. Silvia, "Convolution multipliers and starlike functions", J. Analyse. Math., 41 (1982), 181-192.

[10] H. Silverman, E. M. Silvia and D. N. Telage, "Convolution conditions for convesity", starlikeness and spirallikeness. Math. Z., 162 (1978), 125-130.

Department of Mathematics, Kakatiya University, Warangal-506009, A. P. India.

Department of Mathematics, Kakatiya University, Warangal-506009, A. P. India.

Department of Mathematics, Indian Institute of Technology, Kanpur, (India). 